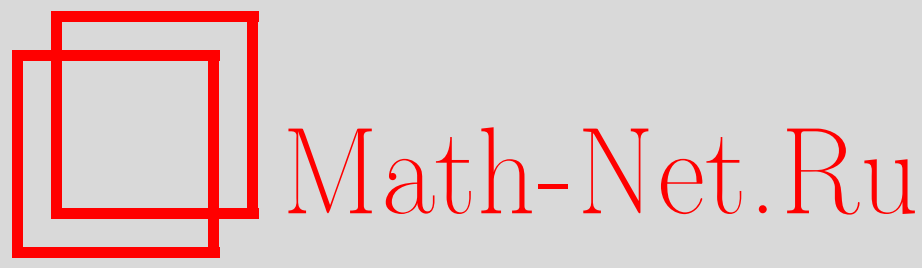

В. Е. Зобов, О показателе степени особой точки производящей функции решеточных деревьев в модели Идена, ТМФ, 2010, том 165, номер 2, 242-256

DOI: https://doi.org/10.4213/tmf6574

Использование Общероссийского математического портала Math-Net.Ru подразумевает, что вы прочитали и согласны с пользовательским соглашением http://www.mathnet.ru/rus/agreement

Параметры загрузки:

IP: 54.157 .27 .8

26 апреля 2023 г., 13:26:25

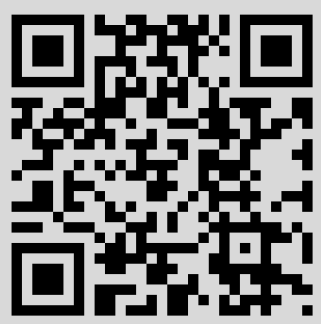




\section{О ПОКАЗАТЕЛЕ СТЕПЕНИ ОСОБОЙ ТОЧКИ ПРОИЗВОДЯЩЕЙ ФУНКЦИИ РЕШЕТОЧНЫХ ДЕРЕВЬЕВ В МОДЕЛИ ИДЕНА}

Рассматривается увеличение числа деревьев при увеличении их размера в модели роста Идена на простых и гранецентрированных гиперкубических решетках при различной размерности пространства. Предлагается уравнение с частными производными первого порядка для производящей функции деревьев, позволяющее связать показатель степени особой точки этой функции с периметром наиболее вероятного дерева. Даются оценки периметров деревьев для рассматриваемых решеток. Теоретические значения показателей степени хорошо согласуются со значениями, полученными ранее методом компьютерного моделирования. Тем самым объясняется близость зависимостей от размерности пространства показателей степеней для простых и гранецентрированных решеток и их отличия от результатов приближения решеток Бете.

Ключевые слова: число решеточных деревьев, периметр дерева, производящая функция, показатель степени особой точки, гиперкубические решетки, решетка Бете, модель Идена.

\section{1. ВВЕДЕНИЕ}

В модели Идена рост кластера происходит путем добавления частиц (узлов, связей) на границе кластера случайно, с равной вероятностью [1]-[6]. Особенно просто это удается сделать на решетке Бете [2], [3], где считается, что каждая новая связь добавляется в новом измерении, поэтому решетка имеет бесконечную размерность. Поскольку не происходит пересечений связей, то полученный кластер является деревом. Для чисел деревьев, зависящих не только от формы, но и от порядка добавления связей, имеем

$$
T_{1}=Z, \quad T_{2}=2 Z(Z-1), \quad T_{n}=\prod_{m=1}^{n}[m(Z-2)+2]=(Z-2)^{n} \frac{\Gamma(n+p+1)}{\Gamma(p+1)}
$$

* Институт физики им. Л. В. Киренского СО РАН, Красноярск, Россия. E-mail: rsa@iph.krasn.ru 
а для производящей функции -

$$
E_{b}(x)=\sum_{n=0}^{\infty} \frac{T_{n} x^{n}}{n !}=\left(1-\frac{x}{x_{b}}\right)^{-1-p},
$$

где $x_{b}=1 / Z_{b}$ - координата особой точки, величина, обратная параметру роста $Z_{b}=Z-2, p=p_{b}=2 /(Z-2)$ - показатель степени особой точки производящей функции, $Z$ - координационное число, $\Gamma(x)$ - гамма-функция. В пределе $n \rightarrow \infty$ формула (1.1) переходит в простое асимптотическое выражение

$$
T_{n} \sim A n !\left(Z_{c}\right)^{n} n^{p}
$$

где в приближении Бете $p=p_{b}, Z_{c}=Z_{b}$ и $A=A_{b}=1 / \Gamma\left(p_{b}+1\right)$.

При построении дерева по тем же правилам на гиперкубической решетке размерности $d$ появляются трудности, если налагается запрет на пересечение ветвей (повторное занятие узлов). Возникающее вследствие этого условия взаимодействие ветвей, получившее название объемного взаимодействия [7], не позволяет найти строгое аналитическое решение задачи, поэтому применяются приближенные методы [1], [3] или моделирование на компьютере [4], [6]. Объемное взаимодействие изменяет свойства деревьев и приводит к их зависимости от вариантов построения деревьев. Обычно в моделях роста интересуются такими свойствами большого кластера, как среднеквадратичный радиус и фрактальная размерность [4], [6]. Усреднение выполняется по сравнительно небольшому числу кластеров. Считается, что в модели Идена вырастает плотный кластер [5], [6].

Интерес представляет исследование свойств всего ансамбля деревьев, заключенных в их производящей функции, введенной выше на примере решетки Бете. Такой анализ оказался необходимым, например, для исследования аналитических свойств временнь́х спиновых корреляционных функций при высоких температурах [8], [9]. Свойства деревьев, полученные после усреднения по всему ансамблю, отличаются от свойств, полученных при усреднении по небольшой выборке. Дело в том, что при построении ансамбля деревьев все возможности будут реализованы, все связи периметра будут последовательно перебраны. Поэтому больший вклад в ансамбль дадут деревья, у которых больше потомков, т. е. больший периметр. Наоборот, при построении выборки из небольшого числа деревьев больший весовой множитель получат деревья с меньшим периметром, поскольку величина периметра стоит в знаменателе при определении вероятности занятия одного узла [5].

Отмеченные свойства ансамбля деревьев должны найти отражение в аналитических свойствах производящей функции. Ранее мы высказали гипотезу о наличии у этой функции особой точки при некотором конечном значении $x_{c}$ переменной $x$. Поскольку мы не можем доказать это утверждение точным расчетом, будем действовать иначе: с одной стороны, проведем численный эксперимент, с другой расчет характеристик особой точки приближенными методами. Совпадение результатов независимых оценок будет свидетельствовать в пользу высказанной гипотезы. Осуществляя эту программу, мы с помощью компьютерного моделирования методом Монте-Карло оценили увеличение полного числа деревьев в ансамбле при 
увеличении числа связей $n$ в дереве для простой (ПК) [10] и гранецентрированной

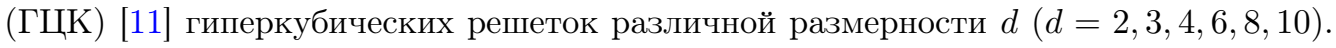
Затем методом разложения по $1 / d$ мы вывели асимптотическую формулу для параметра роста деревьев, определенного как величина, обратная к координате особой точки производящей функции [8], [11]. Получено хорошее согласие теории с результатами численного эксперимента. Из численных данных мы извлекли также показатель степени особой точки производящей функции. Значения показателя для ПК- и ГЦК-решеток оказались близкими между собой и сильно отличающимися от значений для решетки Бете (1.2).

В настоящей работе мы получим теоретическое выражение для этого показателя степени и дадим объяснение его зависимости от размерности пространства. В следующем разделе мы выведем дифференциальное уравнение для производящей функции деревьев. Рост ансамбля деревьев предлагается описывать с помощью наиболее вероятного дерева, для характеристики которого введены два параметра: периметр роста (внешний) и внутренний (мертвый) периметр. Значения этих параметров для ПК- и ГЦК-решеток разной размерности рассчитываются в разделе 3 . В разделе 4 проведено сравнение найденных значений показателя степени с результатами численного моделирования. В приложении выполняется анализ характеристик деревьев на решетке Бете.

\section{2. УРАВНЕНИЕ ДЛЯ ПРОИЗВОДЯЩЕЙ ФУНКЦИИ}

При построении дерева на решетке Бете, которой соответствует бесконечная размерность пространства, каждая новая вершина может быть продолжена в ветвь любой длины. При построении деревьев на гиперкубической решетке конечной размерности при условии запрета на пересечение ветвей часть ветвей не может быть продолжена (мертвые концы), и только часть ветвей, образующих скелет, будет расти. Здесь мы использовали терминологию, предложенную для описания структуры бесконечного кластера в теории перколяции [12]. При переходе от ансамбля $N_{n}$ деревьев с числом связей $n$ к ансамблю $N_{n+1}$ деревьев с числом связей $n+1$ возможны два варианта:

1) связь встраивается в мертвый конец, и периметр не увеличивается;

2) связь встраивается в скелет, и периметр увеличивается (добавленная связь оказывается фактически внутри, а не на конце ветви, поскольку при построении большого дерева добавление связи на конце ветви ведет к сдвигу поверхности, и в результате растет внутренняя часть дерева).

На этом основании периметр $S$ дерева на гиперкубической решетке будем считать состоящим из трех частей:

$$
\nu+m \nu_{1}+m \nu_{2}
$$

где $\nu$ - начальный периметр $(\nu=Z), \nu_{1}$ - часть периметра, приводящая к росту скелета (внешний периметр или периметр роста), $\nu_{2}$ - часть периметра, приводящая к росту мертвых концов (внутренний периметр или мертвый периметр). Для решетки Бете $\nu_{2}=0$, а $\nu_{1}=Z-2$. 
Разделение (2.1) периметра $S$ на три части - это грубое упрощение для передачи качественной картины. Для каждого отдельного дерева может быть свое значение периметра. При строгом подсчете полного числа деревьев в ансамбле следует проводить суммирование по всем значениям периметра:

$$
N_{n+1}=\sum_{S} S N_{n}(S) \cong \sum_{m}\left(\nu+m \nu_{1}+m \nu_{2}\right) N_{n}\left(\nu+m \nu_{1}+m \nu_{2}\right),
$$

где $N_{n}(S)$ - число деревьев из $n$ связей с периметром $S$. При рассмотрении ансамбля больших деревьев эти значения стремятся к некоторым усредненным величинам. Если распределение деревьев по периметру узкое, то прирост обеспечивается деревьями со средними наиболее вероятными параметрами:

$$
N_{n+1} \approx\left(\bar{m} \bar{\nu}_{1}+\bar{m} \bar{\nu}_{2}\right) \sum_{m} N_{n}\left(\nu+m \nu_{1}+m \nu_{2}\right)=\bar{m}\left(\bar{\nu}_{1}+\bar{\nu}_{2}\right) N_{n}
$$

С другой стороны,

$$
N_{n+1}=Z_{c} n N_{n}
$$

Отсюда

$$
Z_{c}=\frac{\bar{m}\left(\bar{\nu}_{1}+\bar{\nu}_{2}\right)}{n}
$$

(в дальнейшем черту сверху будем опускать). Заметим, что подобный подход применялся, например, в теории неупорядоченных систем при определении плотности состояний электронного спектра методом оптимальной флуктуации (наиболее вероятной конфигурации примесей) [12].

Для лучшего понимания предлагаемого описания роста ансамбля деревьев воспользуемся наглядной картиной в виде непрерывной модели. Будем считать два занятых узла связанными, если растояние между ними меньше $r$. Пусть кластер из $n$ связанных занятых узлов характеризуется свободным объемом из $\nu+m \nu_{1}+m \nu_{2}$ доступных узлов. При добавлении нового узла он может попасть во внутренний объем или на поверхность, поэтому число деревьев в ансамбле увеличится в $\nu+m \nu_{1}+m \nu_{2}$ раз. Число попаданий на поверхность равно $\nu+m \nu_{1}$. В этом случае у дерева увеличиваются внутренний объем и поверхность: $\nu+(m+1)\left(\nu_{1}+\nu_{2}\right)$. Число попаданий нового узла во внутренний объем равно $m \nu_{2}$; при этом внутренний объем и поверхность дерева не изменятся.

Для числа деревьев с определенным значением периметра $N_{n}(m) \equiv N_{n}\left(\nu+m \nu_{1}+\right.$ $\left.m \nu_{2}\right)$, растущих по описанным правилам, имеем рекуррентные уравнения

$$
\begin{gathered}
N_{0}(0)=1, \quad N_{1}(1)=\nu N_{0}(0)=\nu \\
N_{2}(1)=\nu_{2} N_{1}(1)=\nu_{2} \nu \\
N_{2}(2)=\left(\nu+\nu_{1}\right) N_{1}(1)=\left(\nu+\nu_{1}\right) \nu
\end{gathered}
$$

в общем виде

$$
N_{n}(m)=\left[\nu+(m-1) \nu_{1}\right] N_{n-1}(m-1)+m \nu_{2} N_{n-1}(m) .
$$


Введем экспоненциальную производящую функцию для деревьев:

$$
F(x, y)=1+\sum_{n=1}^{\infty} \sum_{m=1}^{n} \frac{x^{n} y^{m} N_{n}(m)}{n !}
$$

Домножив обе части уравнения $(2.5)$ на $x^{n} y^{m} / n$ ! и просуммировав, получим уравнение для производящей функции:

$$
F(x, y)=1+\int_{0}^{x}\left[\nu y F\left(x_{1}, y\right)+\nu_{1} y^{2} \frac{\partial}{\partial y} F\left(x_{1}, y\right)+\nu_{2} y \frac{\partial}{\partial y} F\left(x_{1}, y\right)\right] d x_{1} .
$$

Если продифференцировать обе части уравнения (2.7) по $x$, то получим линейное неоднородное уравнение с частными производными первого порядка:

$$
\frac{\partial F}{\partial x}=\left(\nu_{1} y^{2}+\nu_{2} y\right) \frac{\partial F}{\partial y}+\nu y F
$$

Решение уравнения (2.8) найдем методом характеристик [13]:

$$
F(x, y)=\left[1-y \frac{\nu_{1}}{\nu_{2}}\left(e^{\nu_{2} x}-1\right)\right]^{-\nu / \nu_{1}}
$$

Согласно формуле (2.6) параметр $x$ определяет число деревьев, а параметр $y$ - их распределение по периметру. Производящая функция (2.9) как функция от $x$ имеет особую точку при $x=x_{c}$ :

$$
x_{c}=\frac{1}{\nu_{2}} \ln \left(1+\frac{\nu_{2}}{y \nu_{1}}\right) \text {. }
$$

Показатель степени особенности равен

$$
1+p=\frac{\nu}{\nu_{1}}
$$

На основании формул (2.6) и (2.9) для больших деревьев можно приближенно принять

$$
N_{n}=\sum_{m=1}^{n} y^{m} N_{n}(m) \approx x_{c}^{-n} .
$$

Отсюда следует, что $Z_{c}=1 / x_{c}$. Для среднего периметра из формулы (2.12) находим $\bar{m}=\lim _{y=1} \sum_{m=1}^{n} m y^{m} \frac{N_{n}(m)}{N_{n}}=\lim _{y=1} \frac{d}{d y} \ln N_{n} \approx-n \lim _{y=1} \frac{d}{d y} \ln x_{c}=\frac{n}{\left(1+\nu_{1} / \nu_{2}\right) \ln \left(1+\nu_{2} / \nu_{1}\right)}$, и убеждаемся в выполнении формулы (2.4).

Для случая решетки Бете $\nu_{2}=0$ и мы получаем $\bar{m} \approx n$. Легко убедиться, что из (2.10) и (2.11) также получаются известные результаты, если взять $\nu_{1}=Z_{b}=Z-2$ и $\nu=Z$. 


\section{3. ПЕРИМЕТР НАИБОЛЕЕ ВЕРОЯТНОГО ДЕРЕВА}

Поместим начало координат в некоторый узел решетки. Будем записывать координаты других узлов, измеренные в единицах постоянной решетки, в виде

$$
\left(a_{1}, a_{2}, \ldots, a_{d}\right) .
$$

У ПК-решетки ближайшие узлы расположены по координатным осям и имеют в записи (3.1) только одну не равную нулю координату: $a_{i}= \pm 1, i=1, \ldots, d$. У ГЦК-решетки две координаты ближайших узлов в формуле (3.1) отличны от нуля:

$$
a_{i}= \pm \frac{1}{2}, \quad a_{j}= \pm \frac{1}{2}, \quad i, j=1, \ldots, d .
$$

Отсюда для координационных чисел $Z$ находим $Z=2 d$ для ПК и $Z=2 d(d-1)$ для ГЦК.

Для больших деревьев на решетке Бете в приложении найдена полезная характеристика - среднее число ответвлений на одну внутреннюю вершину:

$$
L=\frac{Z-2}{Z-1} .
$$

Следовательно, если $Z$ велико, то у наиболее вероятного дерева чаще других будут встречаться вершины, в которых сходятся три занятые связи (см. рис. 1). Занятые связи будем называть ребрами. На дереве будут встречаться вершины с другим числом ребер, но их меньше. Предположим, что при построении деревьев на гиперкубических решетках рост числа деревьев в ансамбле будет определяться вершинами с тремя ребрами, и определим их вклад в периметр $\nu_{1}$.

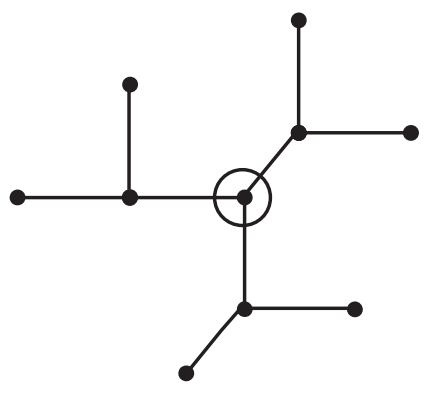

Рис. 1. Фрагмент наиболее вероятного дерева (выделенная вершина обведена кружком).

ПК-решетка. Рассмотрим наиболее вероятное дерево на ПК-решетке размерности $d$. Возьмем какую-нибудь внутреннюю вершину с тремя ребрами, которые будем называть ближними. У такой вершины свободными остаются $Z-3$ связи. Из вершин на концах этих трех ребер выходят по два ребра, которые будем называть дальними. Наиболее вероятна ситуация, при которой эти 6 дальних ребер будут иметь разные направления. Тогда каждому из них окажется параллельной одна из 


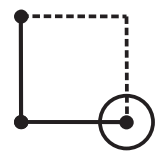

a

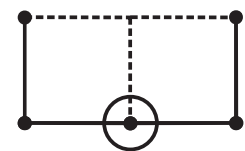

б

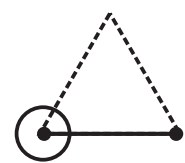

B

Рис. 2. Уменьшение вклада свободной связи в периметр из-за соседства узла к нескольким вершинам дерева на ПК-решетке $\left(\nu_{11}=1 / 2(\mathrm{a}), \nu_{11}=\right.$ $1 / 3$ (б)) и ГЦК-решетке $\nu_{11}=1 / 2($ в)).

свободных связей выделеной вершины. Узел на конце этой связи окажется ближайшим к концу параллельного ребра (см. рис. 2а). Такой узел с равной вероятностью может быть присоединен как к начальной, так и к конечной вершине упомянутого ребра. Поэтому в усредненный периметр выделенной вершины $\nu_{1}$ эти 6 свободных связей войдут с коэффициентом $1 / 2$ :

$$
\nu_{1}=Z-3-\frac{6}{2}=Z-6 .
$$

Найдем поправки порядка $1 / Z$ к полученному значению. Во-первых, направление дальнего ребра может совпасть с направлением ближнего ребра. В этом случае освободится одно из 6 направлений для связей выделенной вершины, т. е. периметр увеличится на $1 / 2$. Вероятность совпадения направлений одного из 6 дальних ребер с одним из 3 ближних ребер равна $6 \cdot 3 / Z$. Соответствующая поправка к периметру (3.3) будет равна $9 / Z$.

Во-вторых, направления двух из 6 дальних ребер могут совпасть между собой. Вероятность такого совпадения равна $12 / Z$. В этом случае освободится одно направление, а у свободной связи начальной вершины, совпавшей по направлению с двумя дальними ребрами (см. рис. 2б), вклад $1 / 2$ в $\nu_{1}$ должен измениться на $1 / 3$. Соответствующая поправка к периметру (3.3) будет равна $4 / Z$.

С учетом поправок получаем

$$
\nu_{1}=Z-6+\frac{13}{Z}
$$

Для трехмерных решеток при $Z=6$ поправка не является малой, и результат (3.4) следует уточнить с учетом конечного числа позиций. Начнем с расположения трех ближних ребер у выделенной вершины. Эти ребра могут быть расположены в одной плоскости или по трем разным координатным осям. Вероятности таких расположений соответственно равны

$$
p_{11}=\frac{1}{d-1}, \quad p_{12}=\frac{d-2}{d-1} .
$$

Возьмем одну из $Z-3=2 d-3$ оставшихся свободными связей у выделенной вершины. Эта связь окажется в новом измерении с вероятностями

$$
p_{21}=\frac{2(d-2)}{Z-3}, \quad p_{22}=\frac{2(d-3)}{Z-3}
$$


в зависимости от расположения близких ребер (3.5). Но она может оказаться на той же оси, что и близкое ребро, с соответствующими вероятностями

$$
p_{31}=1-p_{21}=\frac{1}{Z-3}, \quad p_{32}=1-p_{22}=\frac{3}{Z-3} .
$$

Рассмотрим теперь положение дальних ребер. Если пренебречь запретом на попадание их концов в один и тот же узел, приводящим к поправке следующего порядка малости, то полное число вариантов их расположений будет равно

$$
D^{3}=\left[\frac{(Z-1)(Z-2)}{2}\right]^{3} .
$$

Рассортируем эти варианты по отношению к узлу на конце выбранной свободной связи:

1) нет ребер в ближайшей окрестности; вклад свободной связи в периметр $\nu_{11}=1$;

2) 1 ребро в ближайшей окрестности; вклад свободной связи в периметр $\nu_{11}=1 / 2$;

3) 2 ребра в ближайшей окрестности; вклад свободной связи в периметр $\nu_{11}=1 / 3$;

4) 3 ребра в ближайшей окрестности; вклад свободной связи в периметр $\nu_{11}=1 / 4$.

Оказывается, что число вариантов в таких классах не зависит от выделенных выше способов взаимного расположения ближайших ребер (3.5), но зависит от позиции выбранной свободной связи. Если эта связь направлена в противоположную сторону по той же оси, что одно из ближних ребер, то ребра, выходящие из конца этого ребра, не могут оказаться ближайшими к концу выделенной свободной связи. В этом случае для числа вариантов получаем:

1) нет ребер в ближайшей окрестности; $W_{0}^{\prime}=D[(Z-2)(Z-3) / 2]^{2}$;

2) 1 ребро в ближайшей окрестности; $W_{1}^{\prime}=2 D(Z-2)[(Z-2)(Z-3) / 2]$;

3) 2 ребра в ближайшей окрестности; $W_{2}^{\prime}=D(Z-2)^{2}$.

Если выбранная свободная связь имеет новое направление, то находим, что возможны следующие варианты:

1) нет ребер в ближайшей окрестности; $W_{0}=[(Z-2)(Z-3) / 2]^{3}$;

2) 1 ребро в ближайшей окрестности; $W_{1}=3(Z-2)[(Z-2)(Z-3) / 2]^{2}$;

3) 2 ребра в ближайшей окрестности; $W_{2}=3(Z-2)^{2}[(Z-2)(Z-3) / 2]$;

4) 3 ребра в ближайшей окрестности; $W_{3}=(Z-2)^{3}$.

Для суммарной вероятности двух положений выбранной свободной связи на основании формул (3.5)-(3.7) находим, что если свободная связь направлена в том же измерении, что и ближнее ребро, то

$$
P_{c}=p_{11} p_{31}+p_{12} p_{32}=\frac{3 d-5}{(d-1)(Z-3)} ;
$$

если выбранная свободная связь направлена в новом измерении, то

$$
P_{n}=p_{11} p_{21}+p_{12} p_{22}=\frac{2(d-2)^{2}}{(d-1)(Z-3)} .
$$


Усредненный вклад свободной связи найдем по формуле

$$
\bar{\nu}_{11}=P_{c} \sum_{m=0}^{2} \frac{W_{m}^{\prime}}{(m+1) D^{3}}+P_{n} \sum_{m=0}^{3} \frac{W_{m}}{(m+1) D^{3}} .
$$

Отсюда, умножив на полное число свободных связей у выделенной вершины, найдем ее средний периметр: $\nu_{1}=\bar{\nu}_{11}(Z-3)$.

Квадратная решетка. Формально результат (3.9) примени́м и в случае $d=2$. Однако согласно (3.2) $L=2 / 3$ при $Z=4$, поэтому для повышения точности следует учесть вершины с двумя ребрами и запрет на пересечение дальних ребер. Этот результат можно проинтерпретировать так: если выбрать три внутренние вершины большого дерева, то в среднем из двух вершин будут выходить по три ветви, а из одной - две. Возможные типы окружения таких вершин в случае квадратной решетки показаны на рис. 3 (вершины с четырьмя ветвями учитывать не будем, поскольку, во-первых, они встречаются реже, и, во-вторых, у них не остается свободных связей, поэтому они не дают вклада в $\nu_{1}$ ). Для каждой диаграммы на рисунке приведен ее вклад в периметр $\nu_{1 i}$ и относительный вес $K_{i}$, равный числу расположений на решетке дальних ребер при сохранении положения ближних ребер и топологии данной диаграммы. Усредненный вклад вершины получим, суммируя отдельно вклады вершин с разным числом ответвлений по формуле

$$
\nu_{1}=\frac{1}{3} \frac{\sum_{i} K_{i} \nu_{1 i}}{\sum_{i} K_{i}}+\frac{2}{3} \frac{\sum_{j} K_{j} \nu_{1 j}}{\sum_{j} K_{j}} .
$$

Таким способом находим

$$
\nu_{1}=\frac{1}{3} \frac{62}{57}+\frac{2}{3} \frac{104}{222} \cong 0.67
$$

ГЦК-решетка. Возьмем какую-нибудь внутреннюю вершину с тремя ребрами наиболее вероятного дерева, размещенного на ГЦК-решетке. Выберем одно из трех ребер, выходящих из этой вершины, оканчивающееся в узле $\left(0, \ldots, a_{i}, 0, \ldots\right.$ $\left.\ldots, a_{j}, \ldots, 0\right) \equiv\left(a_{i}, a_{j}\right)$. Для удобства здесь и далее будем писать в формуле (3.1) только отличные от нуля координаты. Ближайшими связями к выбранному ребру будут связи, выходящиеся из начальной вершины и оканчивающиеся в узлах $\left(a_{j}, a_{f}\right)$ и $\left(a_{i}, a_{f}\right)$, где координата $a_{f}$ выбрана из $d-2$ свободных измерений и принимает два значения $\pm 1 / 2$. Следовательно, получаем $\omega=4(d-2)$ ближайшие связи. Узел на конце каждой такой связи является ближайшим и ко второму концу выбранного ребра (рис. 2в), т. е. образуется $\omega$ треугольников из связей вокруг каждого ребра. Такой узел с равной вероятностью может быть присоединен как к начальной, так и к конечной вершине упомянутого ребра. Поэтому относящиеся к таким треугольникам вокруг трех ребер $3 \omega$ свободных связей войдут в усредненный периметр выделенной вершины $\nu_{1}$ с коэффициентом $1 / 2$ :

$$
\nu_{1}=Z-3-\frac{3 \omega}{2}=Z-3-6(d-2) .
$$



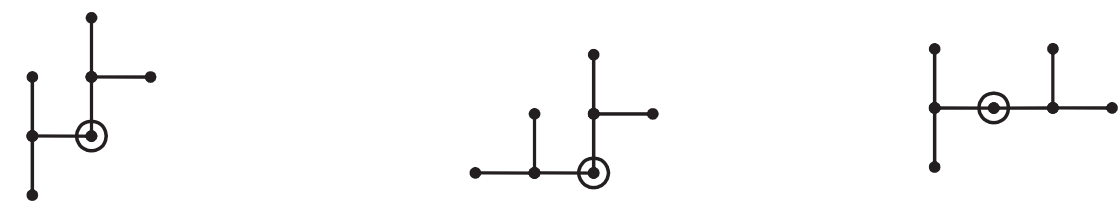

$\nu_{1}=\frac{1}{2}+\frac{1}{2}=1, K=6 \quad \nu_{1}=1+\frac{1}{2}=\frac{3}{2}=1, K=4 \quad \nu_{1}=\frac{1}{2}+\frac{1}{3}=\frac{5}{6}, K=4$

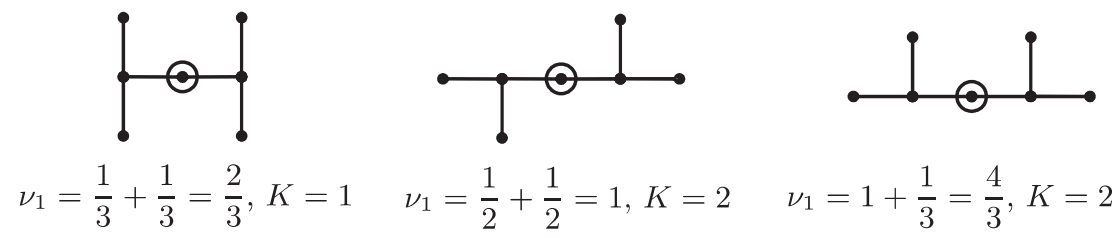

a

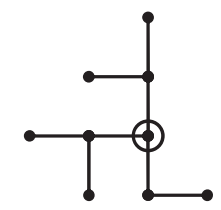

$\nu_{1}=\frac{1}{2}, K=2 \quad \nu_{1}=\frac{1}{2}, K=2$

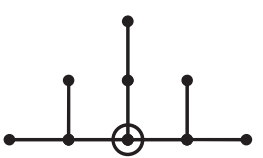

$\nu_{1}=1, K=1$
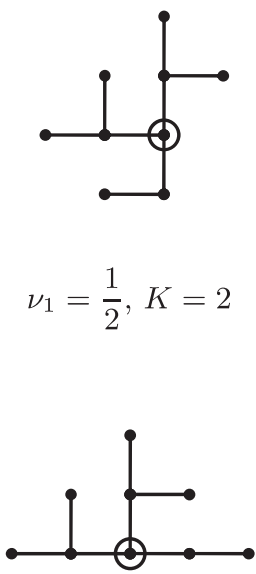

$\nu_{1}=1, K=2$

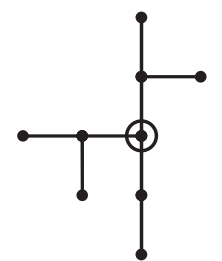

$\nu_{1}=\frac{1}{2}, K=8$

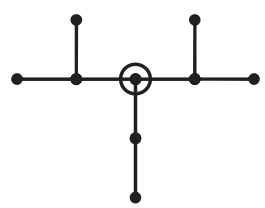

$\nu_{1}=\frac{1}{3}, K=8$

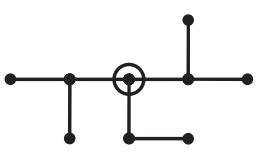

$\nu_{1}=\frac{1}{2}, K=6$

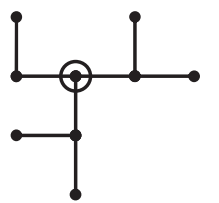

$\nu_{1}=\frac{1}{3}, K=8$

6

Рис. 3. Варианты пространственного расположения на квадратной решетке двух ветвей (а) и трех ветвей (б) вокруг выделенной вершины.

Найдем поправки порядка $1 / Z$ к полученному значению. Во-первых, это поправка от наложения свободных связей двух треугольников, построенных у разных ближних ребер. Во-вторых, это поправки от дальних ребер.

Для оценки поправок первого типа рассмотрим узел в центре, из которого выходит $Z$ пустых связей. Выберем произвольную пустую связь, ведущую в пустой узел с координатами $\left(a_{i}, a_{j}\right)$. Останется $\Omega=Z-1$ пустых связей, в которых можно 
распределить 3 неразличимых ребра $M$ способами:

$$
M=\frac{\Omega(\Omega-1)(\Omega-2)}{6} .
$$

Это множество состояний можно разделить на четыре класса по отношению к выделенной связи:

1) нет ребер, ближайших к выделенной связи; $M_{0}=(\Omega-\omega)(\Omega-\omega-1)(\Omega-\omega-2) / 6$;

2) 1 ребро, ближайшее к выделенной связи; $M_{1}=\omega(\Omega-\omega)(\Omega-\omega-1) / 2$;

3) 2 ребра, ближайших к выделенной связи; $M_{2}=(\Omega-\omega) \omega(\omega-1) / 2$;

4) 3 ребра, ближайших к выделенной связи; $M_{3}=\omega(\omega-1)(\omega-2) / 6$.

Для усредненного вклада одной связи в периметр получаем

$$
\bar{\nu}_{11}=\sum_{m=0}^{3} \frac{M_{m}}{(m+1) M} \text {. }
$$

Отсюда находим периметр как суммарный вклад $Z-3$ пустых связей:

$$
\nu_{1}=\bar{\nu}_{11}(Z-3) \approx Z\left(1-\frac{3}{d}+\frac{11}{2 d^{2}}\right) .
$$

Перейдем к поправкам от дальних ребер. В общем виде это будут ребра, соединяющее узлы $\left(a_{i}, a_{j}\right)$ и $\left(a_{i}, a_{j}, a_{k}, a_{f}\right)$. Перебрав всевозможные расположения ребер, для числа узлов, доступных концу дальнего ребра, находим

$$
V=\frac{Z(d-2)(d-3)}{3}+2 Z(d-2)+2 Z+2 d .
$$

Пусть 3 ближних ребра и 6 дальних ребер располагаются независимо и случайно. Возьмем пустую связь, выходящую из центра, и рассчитаем ее вклад в периметр, уменьшенный из-за попадания ребер в ее ближайшую окрестность.

Сначала распределим 3 ближайших ребра с определенными выше вероятностями $M_{m} / M$ попадания $m$ ребер в область $\omega$. Затем распределим 6 дальних. Полное число расположений 6 неразличимых дальних ребер в области $G=V-4$, оставшейся после расположения близких ребер, будет равно

$$
Q=\frac{G(G-1)(G-2)(G-3)(G-4)(G-5)}{6 !} .
$$

Рассортируем эти состояния по числу попаданий концов дальних ребер в ближайшую окрестность конца рассматриваемой пустой связи, состоящую из $\Omega=Z-1$ узлов. Для усредненного вклада одной связи в периметр получаем

$$
\bar{\nu}_{11}=\sum_{n=0}^{6} \sum_{m=0}^{3} \frac{P_{m n}}{(m+n+1)},
$$

где

$$
P_{m n}=\frac{M_{m}(G-\Omega+m) !(\Omega-m) !}{M Q(G-\Omega+n+m-6) !(\Omega-m-n) !(6-n) ! n !} .
$$


Отсюда находим периметр выделенной вершины как суммарный вклад $Z-3$ пустых связей:

$$
\nu_{1}=\bar{\nu}_{11}(Z-3) \approx Z\left(1-\frac{3}{d}-\frac{7}{2 d^{2}}\right) .
$$

Обратим внимание на предположение о равномерном распределении конца дальнего ребра по области $V$ (3.14). Если рассматривать изолированную систему из двух сочлененных ребер, то вероятность попадания в узел будет зависеть от его расстояния от центра. Хорошо известно, что положение конца длинной цепи без объемных взаимодействий описывается гауссовским распределением. Объемные взаимодействия уменьшают вероятность попадания в центр [7]. Наше равномерное распределение, с одной стороны, упрощает оценки, а с другой стороны, в какой-то степени учитывает влияние объемного взаимодействия на структуру наиболее вероятного дерева.

\section{4. РАСЧЕТ ПОКАЗАТЕЛЯ СТЕПЕНИ И ОБСУЖДЕНИЕ РЕЗУЛЬТАТОВ}

Показатель степени особой точки производящей функции определен через средний периметр формулой (2.11). Зависимость $p$ от $d$, рассчитанная по формулам (3.12) и (3.16), показана на рис. 4 вместе с результатами, полученными нами ранее на основе данных численного моделирования методом Монте-Карло [10], [11]. При $d=2$ взято найденное выше значение $\nu_{1} \cong 0,67$ (3.10), приводящее к $p \approx 5$. Мы наблюдаем хорошее согласие теоретических оценок с результатами численного эксперимента. Вместе с тем наблюдается существенное расхождение этих значений показателя со значениями в приближении решетки Бете (1.2)

Другим важным параметром, определяющим рост числа деревьев в ансамбле, служит параметр роста $Z_{c}$. Его зависимость от $d$ была изучена нами в предыдущих работах [8]-[11] для ПК- и ГЦК-решеток. Результаты численного моделирования и $1 / d$-разложения находятся в хорошем согласии. В предложенной выше модели параметр роста определяет формула (2.10). Предположим, что $Z=\nu=\nu_{1}+\nu_{2} / c+\delta$, и преобразуем формулу (2.10):

$$
\frac{Z}{Z_{c}}=(1+p) \frac{\ln (1+a)}{a},
$$

где $a=\nu_{2} / \nu_{1}=c p(1-\delta / Z)-c \delta / Z$. В случае решеток Бете $\nu=Z, \nu_{2}=0, \nu_{1}=Z_{c}=$ $Z-2$ и $\delta=2$. Результаты численного эксперимента для гиперкубических решеток показали, что $\delta>2,2>c>1$. Физический смысл параметра $c$ заключается в том, что при построении всевозможных деревьев ансамбля связи, входящие в $\nu_{2}$, могут быть встроены в деревья по-разному, например, могут проходиться ветвью в противоположных направлениях, т. е. двумя способами.

Приближенное выражение для показателя степени при $d \gg 1$ получаем после подстановки асимптотических выражений для периметра $\nu_{1}$. Для ПК-решетки из формулы (3.4) получаем

$$
p=\frac{3-13 / 4 d}{d-3+13 / 4 d},
$$




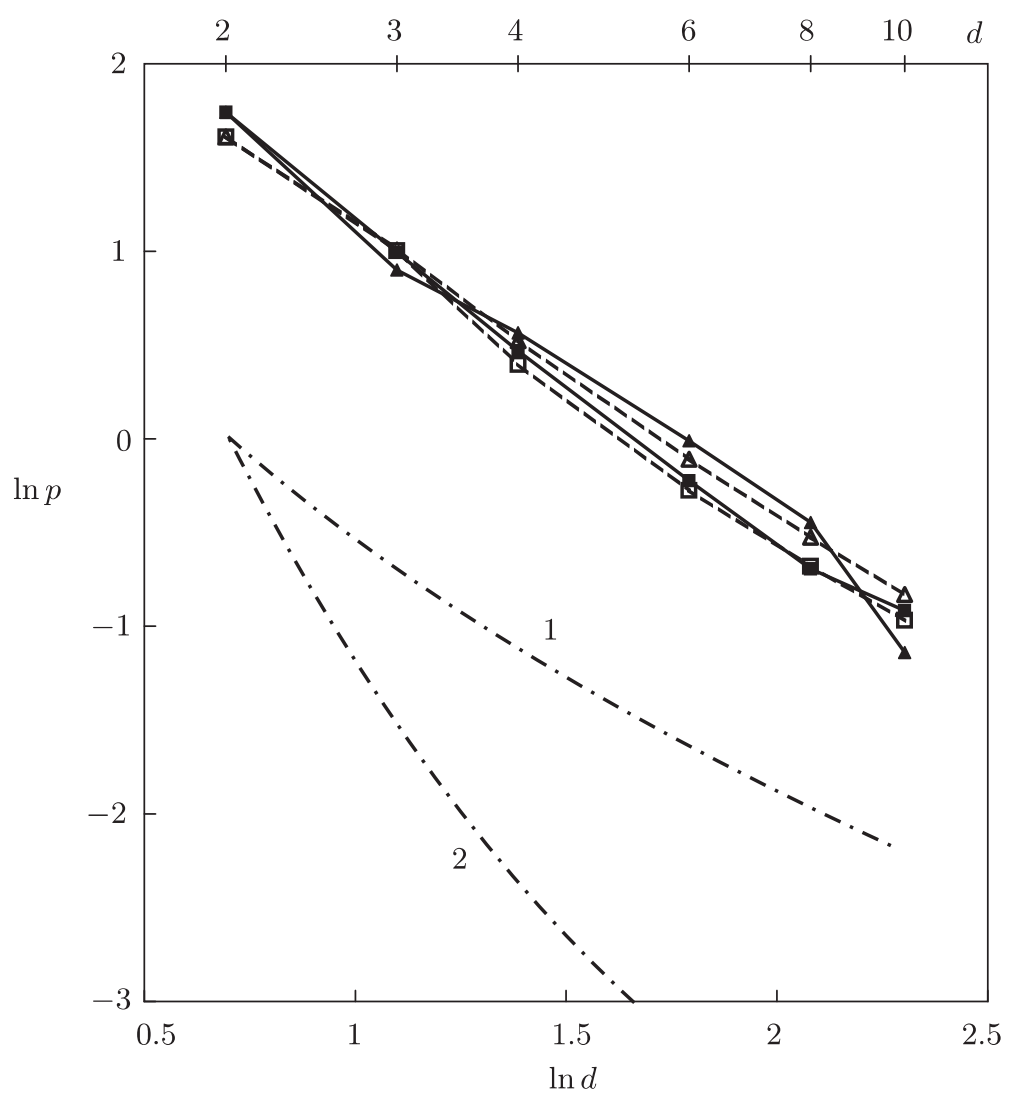

Рис. 4. Зависимости показателей степени от размерности пространства для ПК-решеток (квадраты) и ГЦК-решеток (треугольники): $\mathbf{\square}, \mathbf{\Delta}$, сплошные линии - результаты численного эксперимента [10], [11]; $\square, \triangle$, штриховые линии - расчет по полученным формулам. Штрих-пунктирные линии соответствуют результатам приближения Бете: 1 - для ПК-решеток, 2 - для ГЦК-решеток.

для ГЦК-решетки из формулы (3.18) -

$$
p=\frac{3+7 / 2 d}{d-3-7 / 2 d} .
$$

Показатели (4.2) и (4.3) совпадают с точностью до линейных по $1 / d$ поправок. Квадратичные поправки получились разными. Недостаточная точность численного эксперимента (20\%), которая связана с ограничением размера доступных для моделирования деревьев, не позволяет подтвердить или опровергнуть эти небольшие различия. С другой стороны, причиной несовпадения теоретических результатов (4.2) и (4.3) может оказаться неточность в определении наиболее вероятного дерева, используемого при расчете усредненного периметра $\nu_{1}$. 
Для ПК- и ГЦК-решеток в приближении Бете после подстановки значений $Z$ находим соответственно

$$
p_{b}=\frac{1}{d-1}, \quad p_{b}=\frac{1}{d(d-1)-1} .
$$

Принципиальное отличие двух подходов состоит в способе учета неограниченно растущих ветвей, выходящих из вершины. В случае приближения решеток Бете, как уже говорилось выше, таковыми являются все $Z-1$ ветвей. Независимость растущих ветвей нашла отражение в структуре уравнений (П.2) и (П.3) (см. ниже в приложении) для производящих функций, тогда как на гиперкубических решетках вследствие запрета на пересечение ветвей только небольшая их часть может расти неограниченно. Для получения возможности учета объемного взаимодействия ветвей мы подошли к выводу уравнения для производящей функции не через анализ роста отдельных веток, а через анализ роста всего периметра. Новая форма уравнения для производящей функции (2.8) позволила в нашем подходе свести проблему учета объемного взаимодействия к определению локальных свойств окрестности вершины наиболее вероятного дерева и объяснить, в конечном счете, численный эксперимент.

\section{ПРИЛОЖЕНИЕ}

\section{Характеристики деревьев на решетке Бете}

Рассмотрим решетку Бете с координационным числом $Z$. Наряду с производящей функцией деревьев (1.2) будем использовать производящую функцию так называемых деревьев с висячим корнем, у которых к корню присоединяется только одна связь:

$$
E_{1}(x)=\left(1-\frac{x}{x_{b}}\right)^{-1 /(Z-2)} .
$$

В приближении Бете построение различных ветвей дерева проводится независимо, поэтому производящая функция (П.1) удовлетворяет самосогласованному уравнению

$$
E_{1}(x)=1+\int_{0}^{x}\left(E_{1}(x)\right)^{q} d x_{1},
$$

где $q=Z-1$. Введем функцию $E_{q}(x)=\left(E_{1}\left(x_{1}\right)\right)^{q}$, для которой из (П.2) получаем уравнение

$$
E_{q}(x)=1+\sum_{k=1}^{q}\left(\begin{array}{l}
q \\
k
\end{array}\right)\left[\int_{0}^{x} E_{q}\left(x_{1}\right) d x_{1}\right]^{k},
$$

представленное в виде суммы по числу ветвей $k$, выходящих из вершины. Добавим под знаком суммы весовой множитель числа ответвлений $b^{k-1}$ :

$$
E_{q}(x)=\sum_{k=0}^{q} b^{k-1}\left(\begin{array}{l}
q \\
k
\end{array}\right)\left[\int_{0}^{x} E_{q}\left(x_{1}\right) d x_{1}\right]^{k} .
$$

Введем функцию

$$
Y=1+b \int_{0}^{x} E_{q}\left(x_{1}\right) d x_{1} .
$$


Для функции $Y$ из (П.4) получаем уравнение

$$
\frac{d Y}{d x}=Y^{q}-a
$$

где $a=1-b$. Его решение может быть записано в виде

$$
x=\int_{1}^{Y} \frac{d y}{y^{q}-a} .
$$

Отсюда для координаты особой точки находим

$$
x_{c}=\int_{1}^{\infty} \frac{d y}{y^{q}-a}, \quad \frac{d}{d b} x_{c}=-\int_{1}^{\infty} \frac{d y}{\left(y^{q}-a\right)^{2}} .
$$

В частности, при $b=1(a=0)$

$$
x_{c}=\frac{1}{Z-2}, \quad \frac{d}{d b} x_{c}=-\frac{1}{2 Z-3} .
$$

Введем две усредненные характеристики деревьев с $n$ связями: среднее число ответвлений $B$ и среднее число ответвлений на один внутренний узел $L$, которые будем определять по следующим формулам:

$$
B=\frac{d}{d b} \ln T_{n}(b) \approx-n \frac{d}{d b} \ln x_{c}, \quad L=\frac{B}{n-B-1} .
$$

В частности, при $b=1(a=0)$

$$
B=n \frac{Z-2}{2 Z-3}, \quad L=\frac{Z-2}{Z-1} .
$$

\section{Список литературы}

[1] G. Parisi, Y.-C. Zhang, Phys. Rev. Lett., 53:19 (1984), 1791-1794.

[2] J. Vannimenus, B. Nickel, V. Hakim, Phys. Rev. B, 30:1 (1984), 391-399.

[3] R. Friedberg, Ann. Phys., 171:2 (1986), 321-363.

[4] Z. Rácz, M. Plischke, Phys. Rev. A, 31:2 (1985), 985-994.

[5] D. Dhar, Phys. Rev. Lett., 54:18 (1985), 2058.

[6] L. R. Paiva, S. C. Ferreira Jr., J. Phys. A, 40:1 (2007), F43-F49.

[7] А.Ю. Гросберг, А.Р. Хохлов, Статистическая физика макромолекул, Наука, М., 1989.

[8] В. Е. Зобов, ТМФ, 123:1 (2000), 116-131.

[9] В. Е. Зобов, М. А. Попов, ТМФ, 136:3 (2003), 463-479.

[10] В. Е. Зобов, М. А. Попов, ТМФ, 126:2 (2001), 325-336.

[11] В. Е. Зобов, М. А. Попов, ТМФ, 144:3 (2005), 564-576.

[12] Б. И. Шкловский, А.Л. Эфрос, Электронные свойства легированных полупроводников, Наука, М., 1979.

[13] В. И. Смирнов, Курс высшей математики. Т. 2, 4, Гостехиздат, М., 1953.

Поступила в редакцию 5.03.2010, после доработки 21.05.2010 